\title{
Article
}

http://dx.doi.org/10.11646/phytotaxa.173.1.2

\section{New synonyms and combinations in Eurasian Brassicaceae (Cruciferae)}

\author{
DMITRY A. GERMAN
}

Centre for Organismal Studies, Heidelberg University, Im Neuenheimer Feld 345, 69120 Heidelberg, Germany; \& South-Siberian Botanical Garden, Altai State University, Lenina str. 61, 656049 Barnaul, Russia

E-mail: oreoloma@rambler.ru

\begin{abstract}
Taxonomy of miscellaneous taxa of Eurasian Brassicaceae is updated. Eight names at the species rank are reduced for the first time to synonymy of what follows them in parentheses: Cardamine szeewaldiana (C. macrophylla), Hesperis hyrcanica (Clastopus vestitus), Arabis oxyota (Crucihimalaya bursifolia), Hesperis speciosa and Parrya integerrima (Dendroarabis fruticulosa), Cheiranthus taraxacifolius (Erysimum cuspidatum), Draba ishkomania (Scapiarabis saxicola), Velarum tzvelevii (Sisymbrium officinale). Three new combinations (Hormathophylla kuepferiana, Isatis odontogera, and Parrya khorasanica) are proposed, first of them as a replacing name for a later homonym in the genus Hormathophylla. Three names (Arabis oxyota, Clastopus vestitus, and Crucihimalaya bursifolia) are lectotypified (for $C$. vestitus, a previous choice is narrowed) and a neotype is designated for Isatis odontogera. Nomenclatural comments are provided where needed.
\end{abstract}

\section{Introduction}

The work on taxonomy and nomenclature of different groups of predominantly North Eurasian Brassicaceae (Cruciferae) regularly reveals various nomenclatural items requiting clarification. Among the most common problems are the old and sometimes newly appearing species names with uncertain taxonomical identity ("unresolved"). Identification and proper taxonomic attribution of such names is necessary in order to clarify the taxonomy of relevant groups. The present communication deals with eight such names in the Brassicaceae which are recognized as new synonyms of the seven species from different genera of Eurasian mustards. Apart, three species nomenclature of which needs to be updated, are also included.

\section{Materials and methods}

The collections of Brassicaceae deposited at the herbaria B, BP, GOET, HAL, LE, MW, P, and W were examined. Materials of G, K, and partly P available online (CGH 2014, The Herbarium Catalogue 2006, Muséum national...) were also consulted along with the digital photos obtained from BUCA and ERE. Relevant literature including all sources containing protologues was studied and used for making taxonomic interpretations. Floras and some global databases (The Plant List 2013, Tropicos.org) were also consulted. References to the International Code of Nomenclature for algae, fungi and plants are provided where needed according to its Melbourne edition (McNeill et al. 2012).

\section{Results}

The following eight new synonyms were established and three new combinations were proposed. 
Parrya khorasanica (Rech.f. \& Aellen) D.A. German \& Al-Shehbaz, comb. nov. $\equiv$ Hesperis khorasanica Rech.f. \& Aellen in Rechinger (1951: 65). Holotype:-IRAN. Prov. Khorasan: [in declivibus saxosis] Montes Hazar Masdjid. 2000-2500 m. 8-9. VI. 1948. K.H. Rechinger 5062, [E. Esfandiari] \& P. Aellen (W!); isotype in IRAN (IRAN No. 5093) (Termeh, 1996).

While transferring all species of Pseudoclausia Popov (1955: 18) to the expanded Parrya (Al-Shehbaz \& German 2013), Pseudoclausia turkestanica (Lipsky 1904: 41) Vassiljeva (1961: 244) was given a name Parrya lipskyi D.A. German \& Al-Shehbaz in Al-Shehbaz \& German (2013: 463) to avoid creation of a later homonym of Parrya turkestanica (Korshinsky 1898: 407) Busch (1939: 644). Unfortunately, a taxonomic synonym Hesperis khorasanica was overlooked which has priority in Parrya over Parrya lipskyi.

\section{Acknowledgements}

I cordially thank curators of all cited herbaria for providing the opportunity of working with the material. I am also deeply grateful to Antonina Ph. Ilyinska for help with some literature and search of material on Isatis odontogera in KW; to Vandika E. Avetisian for fruitful discussion of certain taxonomic questions and sending the image of the here designated neotype of I. odontogera from ERE; to Eckhard von Raab-Straube and Sarah Bollendorff for help in studying some specimens in B; to Christine Bartram for thorough search of type material of Parrya integerrimal Hesperis speciosa in CGE; to Laszlo Bartha for help in search of the original material of Cardamine szeewaldiana and to Sorin Stefanut for kind sending the images of the type of this taxon; to Amir Sultan for confirmation of availability of the type of Draba ishkomania in RAW; and to Alexander N. Sennikov for consultations on some nomenclatural questions.

\section{References}

Aiken, S.G., Dallwitz, M.J., Consaul, L.L., McJannet, C.L., Boles, R.L., Argus, G.W., Gillett, J.M., Scott, P.J., Elven, R., LeBlanc, M.C., Gillespie, L.J., Brysting, A.K., Solstad, H. \& Harris, J.G. (2007) Flora of the Canadian Arctic Archipelago: Descriptions, Illustrations, Identification, and Information Retrieval. NRC Research Press, National Research Council of Canada, Ottawa. Available from: http://nature.ca/aaflora/data (accessed 07 Mar 2014).

Al-Shehbaz, I.A. (2005) Nomenclatural notes on Eurasian Arabis (Brassicaceae). Novon 15(4): 519-524.

Al-Shehbaz, I.A. (2012) A generic and tribal synopsis of the Brassicaceae (Cruciferae). Taxon 61(5): 931-954.

Al-Shehbaz, I.A. \& German, D.A. (2013) A synopsis of the genus Parrya (Brassicaceae). Kew Bulletin 68(3): 457-475. http://dx.doi.org/10.1007/s12225-013-9450-4

Al-Shehbaz, I.A. \& Mulligan, G.A. (2013) New or noteworthy species of Draba (Brassicaceae) from Canada and Alaska. Harvard Papers in Botany 18(2): 101-124.

http://dx.doi.org/10.3100/025.018.0203

Al-Shehbaz, I.A. \& O'Kane, S.L., Jr. (2003) Transberingia, a new generic name replacing the illegitimate Beringia (Brassicaceae. Novon 13(4): 396.

http://dx.doi.org/10.2307/3393368

Berkutenko, A.N. (1983) Krestotsvetnye Kolymskogo nagor'ya [Cruciferae of the Kolyma upland]. Academy of Sciences of the USSR, Vladivostok, 164 pp. (In Russian)

Boczantzeva, V.V. (1979) The new genus Galitzkya V. Boczantzeva (Cruciferae). Botanicheskii Zhurnal (Moscow \& Leningrad) 64(10): 1440-1442. (In Russian)

Boissier, E. (1867) Flora Orientalis 1. H. Georg, Basel \& Genève, XXXIV + 1017 pp.

Bordzilowski, E.I. (1935) New data on the flora of Caucasus. Journal de l'Institut botanique de l'Académie des Sciences de la RSS d'Ukraine 3(2): 69-84. (In Ukrainian)

Bordzilowski, E. (1938) De plantis novis et rarioribus e Transcaucasia. Symposium dedicated to the memory of A.V. Fomin. Inst. Bot. Acad. Sci. URSR, Kiev, pp. 58-65. (In Russian)

Bornmüller, J. (1906) Die Arten und Formen der persischen Cruciferengattungen Clastopus Bge. und Straussiella Hausskn. Repertotium novarum specierum regni vegetabilis 3 (33-34): 114-116. 
http://dx.doi.org/10.1002/fedr.19060033306

Botschantzev, V.P. (1959) De Cuciferis notae criticae, III. Botanicheskie Materialy Gerbariya Botanicheskogo Instituta V.L. Komarova Akademii Nauk SSSR 19: 105-108. (In Russian)

Botschantzev, V.P. (1968) De Cruciferis notae criticae, 6. Novosti Sistematiki Vysshikh Rastenii [Novitates Systematicae Plantarum Vascularium] 5: 140-146. (In Russian)

Botschantzev, V.P. (1972) On Parrya R. Br., Neuroloma Andrz. and some other genera (Cruciferae). Botanicheskii Zhurnal (Moscow \& Leningrad) 57(6): 664-673. (In Russian)

Brown, R. (1823) Chloris melvilliana. W. Clowes, London, 52 [+ 4 plates] pp.

Busch, N.A. (1939) Cruciferae B. Juss. In: Komarov, V.L. (ed.) Flora of USSR 8. Publishers of Academy of Sciences of USSR, Moscow \& Leningrad, pp. 14-606. (In Russian)

Candolle, A.P. de (1821) Regni vegetabilis systema naturale 2. Socii Treuttel et Würtz, Parisiis [Paris], [II] + 745 pp.

CHG (2014) Catalogue des herbiers de Genève. Conservatoire \& Jardin botaniques de la Ville de Geneve. Available from: http://www. ville-ge.ch/musinfo/bd/cjb/chg (accessed 28 Apr 2014).

Cullen, J. (1965) Erysimum L. In: Davis, P.H. (ed.). Flora of Turkey and the East Aegean Islands 1. [Edinburgh] University Press, Edinburgh, pp. 466-478.

Czerepanov, S.K. (1995) Vascular plants of Russia and adjacent states (the former USSR). Cambridge University Press, Cambridge, X + $519 \mathrm{pp}$.

Desvaux, N.A. (1815) Sur la famille des Plantes Crucifères. Journal de Botanique appliqué, à l'Agriculture, à la Pharmacie, à la Medecine et aux Arts 3(4): 145-187.

Don, G. (1831) A General History of the Dichlamydeous Plants 1. J. G. \& F. Rivington, London, XXVIII + 818 pp.

Dorofeyev, V.I. (2012) Brassicaceae Burnett (Cruciferae Juss.). In: Tzvelev, N.N. (ed.) Conspectus florae Europae orientalis 1. KMK Scientific Press, St. Petersburg \& Moscow, pp. 364-437. (In Russian)

Dudley, T.R. \& Cullen J. (1965) Studies in the Old World Alysseae Hayek. Feddes Repertorium 71(1-3): 218-228. http://dx.doi.org/10.1002/fedr.19650710105

Edgeworth, M.P. (1846) Descriptions of some unpublished species of plants from North-West India. Transactions of the Linnean Society of London 20(1): 23-92.

Euro+Med (2006-): Euro+Med PlantBase - the information resource for Euro-Mediterranean plant diversity. Available from: http://ww2. bgbm.org/EuroPlusMed (accessed 27 Apr 2014).

Fawcett, W. \& Rendle, A.B. (1914) Flora of Jamaica 3. British Museum, London, XXIV + $280+[10]$ pp.

Fischer, F.E.L. \& Meyer, C.A. (1835) Index seinum, quae hortus botanicus Imperialis petropolitanus pro mutua commutatione offert. Accedunt animadversiones botanicae nonnullae. Petropoli [St. Petersburg], 42 pp.

Fischer, F.E.L. \& Meyer, C.A. (1842) Enumeratio altera plantarum novarum a cl. Schrenk lectarum. Typis G. Fischeri, Petropoli [St. Petersburg], 77 pp.

German, D.A. (2005a) Contribution to the taxonomy of Arabidopsis s. 1. The status of Transberingia and two new combinations in Crucihimalaya (Cruciferae). Turczaninowia 8, 4: 5-15.

German, D.A. (2005b) Type specimens of Altai Cruciferae taxa described in 1829-1841 by C.A. Meyer, A.A. Bunge and C.F. Ledebour deposited in the Herbarium of the Komarov Botanical Institute (LE). Novosti Sistematiki Vysshikh Rastenii [Novitates Systematicae Plantarum Vascularium] 37: 233-267. (In Russian).

German, D.A. (2011) Typification of selected names of Cruciferae taxa from Siberia and some neighboring regions. Turczaninowia 14, 1: 45-54.

German, D.A. \& Al-Shehbaz, I.A. (2008) Dendroarabis, a new Asian genus of Brassicaceae. Harvard Papers in Botany 13(2): $289-291$. http://dx.doi.org/10.3100/1043-4534-13.2.289

Ikonnikov-Galitzky, N.P. (1936) A new species of the fam. Cruciferae in the Mongolian flora. Trudy Botanicheskogo Instituta Akademii Nauk SSSR, Ser. 1. Flora i Sistematika Vysshikh Rastenii [Acta Instituti Botanici Academiae Scientarum URSS, Ser. 1. Flora et Systematica Plantarum Vascularium] 3: 189-193. (In Russian).

Jackson, B.D. (1893) Index Kewensis: an enumeration of the genera and species of flowering plants 1. Clarendon Press, Oxford, 1268 pp.

Jackson, B.D. (1895) Index Kewensis: an enumeration of the genera and species of flowering plants 2. Clarendon Press, Oxford, 1299 pp.

Kamelin, R.V. (1973) Florogenetic analysis of the natural flora of mountainous Middle Asia. Science Press, Leningrad, 356 pp.

Khatri, K.S. (1990) Cardamine L. sect. Macrophyllum O.E. Schulz (Cruciferae Juss.) in the Asian territory of the Soviet Union. A morphological, anatomical and phylogenetical study with taxonomic revision. Folia Geobotanica et Phytotaxonomica 25(2): 197216.

Koch, M.A., Karl, R., German, D.A. \& Al-Shehbaz, I.A. (2012) Systematics, taxonomy and biogeography of three new Asian genera from 
the Brassicaceae tribe Arabideae: an ancient distribution circle around the Asian high mountains. Taxon 61(5): 955-969.

Korshinsky, S. (1898) Fragmenta florae Turkestanicae. Plantae novae vel minus cognitae Turkestaniae describuntur. Bulletin de l'Académie Impériale des sciences de Saint-Pétersbourg ser. 5, 9: 399-424.

Kotov, M.I. (1979) Brassicaceae (Cruciferae). In: Fedorov, An.A. \& Gusev, Yu.D. (eds.) Flora partis Europaeae URSS 4. Science Press, Leningrad, pp. 30-148. (In Russian)

Küpfer, P. (1974) Recherches sur les liens da parenté entre la flora orophile des alpes et celle des Pyrénées. Boissiera 23: 5-322.

Lamarck, J.B. (1789) Encyclopédie méthodique: botanique 3. Panckoucke, Paris \& Plomteux, Liége, VIII + 759 pp.

Ledebour, C.F. (1841) Flora Rossica sive enumeratio plantarum in totius imperii rossici provinciis europaeis, asiaticis et americanis hucusque observatarum 1(1). Sumptibus librariae E. Schweizerbart, Stuttgartiae [Stuttgart], [I]-XVI + [1]-240 pp. http://dx.doi.org/10.5962/bhl.title.66688

Linnaeus, C. (1753) Species plantarum. L. Salvius, Holmiae [Stockholm], 1200 pp.

Lipsky, V.I. (1904) Contributio ad floram Asiae Mediae. II. Trudy Imperatorskogo S.-Peterburgskogo Botanicheskogo Sada [Acta Horti Petropolitani] 23: 1-247.

Marschall von Bieberstein, F.A. (1798) Tableau des provinces situées sur la côte occidentale de la mer Caspienne entre les fleuves Terek et Kour. St. Petersbourg, 120 pp.

McNeill, J., Barrie, F.R., Buck, W.R., Demoulin, V., Greuter, W., Hawksworth, D.L., Herendeen, P.S., Knapp, S., Marhold, K., Prado, J., Prud'homme van Reine, W.F., Smith, G.F., Wiersema, J.H. \& Turland, N.J. (eds.) (2012) International Code of Nomenclature for algae, fungi, and plants (Melbourne Code). Adopted by the Eighteenth International Botanical Congress Melbourne, Australia, July 2011. Koeltz Scientific Books, Königstein, XXX + 208 pp. [Regnum Vegetabile 154].

Meyer, C.A. (1831) Classis Tetradynamia. In: Ledebour, C.F., Meyer, C.A. \& Bunge A.A. Flora Altaica 3. Typis et Impensis G. Reimeri, Berolini [Berlin], pp. 1-219.

Muséum national d'Histoire naturelle, Paris (France) Collection: Vascular plants (P). Available from: http://science.mnhn.fr/institution/ mnhn/search (accessed 30 Apr 2014).

Pallas, P.S. (1771) Reise durch verschiedene Provinzen des Rußischen Reichs 1. Imp. Acad. Wiss., St. Petersburg, 504 pp.

Pallas, P.S. (1794) Voyages de Professeur Pallas dans plusieurs provinces de l'Empire de Russie et dans l'Asie septentrionale. Traduits de l'Allemand parle C. Gauthier de la Peyronie. 8. Appendix. Chez Maradan, Paris, 463 pp. http://dx.doi.org/10.5962/bhl.title.63438

Polatschek, A. (2010) Revision der Gattung Erysimum (Cruciferae): Teil 1: Russland, die Nachfolgestaaten der USSR (excl. Georgien, Armenien, Azerbaidzan), China, Indien, Pakistan, Japan und Korea. Annalen des Naturhistorischen Museums in Wien, B 111: 181275.

Popov, M.G. (1955) 3821a. Clausia aprica (Steph.) K.-Trotz. Spisok Rastenii Gerbariya Flory SSSR [Schedae ad Herbarium Florae URSS] 13: 18-19.

Price, R.A., Al-Shehbaz, I.A. \& O’Kane, S.L., Jr. (2001) Beringia (Brassicaceae), a new genus of Arabidopsoid affinity from Russia and North America. Novon 11(3): 332-336. http://dx.doi.org/10.2307/3393041

Prodan, I. (1927) Contribuțiuni la Flora Siberiei / Beiträge zur Flora von Sibirien. Buletinul Grădinii Botanice și al Muzeuli Botanic de la Universitatea din Cluj 18(6): 103-124.

Rechinger, K.H. (1951) Cruciferae iranicae novae vel minus cognitae. Phyton (Horn) 3(1-2): 44-68.

Rechinger, K.H. (1968a) Clastopus. In: Rechinger, K.H. (ed.) Flora Iranica 57. Akademische Druck- u. Verlagsanstalt, Graz, pp. 139140.

Rechinger, K.H. (1968b) Koeiea. In: Rechinger, K.H. (ed.) Flora Iranica 57. Akademische Druck- u. Verlagsanstalt, Graz, pp. 247-248, 349.

Regel, E. (1862). Aufzaehlungder von Radde in Baikalien, Dahurien und am Amur sowie der vom Herrn von Stubendorff auf seiner Reise durch Sibirien nach Kamtschatka, und der von Rieder, Kussmisscheff und anderen in Kamtschatka gesammelten Pflanzen. I. Abteilung. Dicotyledoneae. Polypetalae. Bulletin de la Société Impériale des Naturalistes de Moscou 34(3): 1-211.

Ross, R. (2002) Which illustrations are original material? Taxon 51(4): 523-524. http://dx.doi.org/10.2307/1554867

Ruprecht, F.J. (1869) Flora Caucasi. Pars 1. Mémories de l'Académie Imperiale des Sciences de St.-Pétersbourg, 7 sér., 15 (2): [I-IV] + $1-302$.

Schulz, O.E. (1903) Monographie der Gattung Cardamine. Botanische Jahrbücher für Systematik, Pflanzengeschichte und Pflanzengeographie 32(2-3): 280-416.

Scopoli, J.A. [G.A.] (1772) Flora carniolica exhibens plantas Carnioliae indigenas et distributas in classes, genera, species, varietates, ordine linnaeano 2. Editio secunda aucta et reformata. Impensis Joannis Pauli Krauss, Bibliopolae Vindobensis [Vienna], pp. [ILXXII] $+[1]-448+[1-16]+$ pl. 33-65. 
Sprengel, C. (1825) Caroli Linnaei systema vegetabilium. Vol. 2. Classis 6-15. Sumptibus Librariae Dieterichianae, Gottingae [Göttingen], $939 \mathrm{pp}$.

http://dx.doi.org/10.5962/bhl.title.822

Stafleu, F.A. \& Cowan, R.S. (1976) Taxonomic literature: a selective guide to botanical publications and collections with dates, commentaries and types. Volume I: A-G. Ed. 2. Bohn, Scheltema \& Holkema, Utrecht, [XL] + 1136 pp. [Regnum Vegetabile 94]

http://dx.doi.org/10.5962/bhl.title.48631

Stafleu, F.A. \& Cowan, R.S. (1979) Taxonomic literature: a selective guide to botanical publications and collections with dates, commentaries and types. Volume II: H-Le. Ed. 2. Bohn, Scheltema \& Holkema, Utrecht; Dr. W. Junk b.v., Publishers, The Hague, [Antwerpen], XVIII + 1136 pp. [Regnum Vegetabile 98].

http://dx.doi.org/10.5962/bhl.title.48631

Stafleu, F.A. \& Cowan, R.S. (1986) Taxonomic literature: a selective guide to botanical publications and collections with dates, commentaries and types. Volume IV: Sti-Vuy. Ed. 2. Bohn, Scheltema \& Holkema, Utrecht; Dr. W. Junk b.v., Publishers, The Hague, [Antwerpen]; Boston, 926 pp. [Regnum Vegetabile 115].

http://dx.doi.org/10.5962/bhl.title.48631

Steudel, E.G. (1841) Nomenclator botanicus 2. Ed. 2. Typis et Sumptibus I. G. Cottae, Stuttgartiae et Tubingae [Stuttgart \& Tubingen], $810 \mathrm{pp}$.

Sweet, R. ([1831-]1833). Hortus britannicus 5. Ed. 2. James Ridgway, London, VI pp. + pl. 101-200 + [7] pp.

Termeh, F. (1996) Type specimens of K.H. Rechinger in the Herbarium Ministerii Iranici Agriculturae. Annalen des Naturhistorischen Museums in Wien, B 98 (Suppl.): 117-125.

The [Kew] Herbarium Catalogue (2006). Available from: http://www.kew.org/herbcat (accessed 14 Mar 2014).

The Plant List (2013) Version 1.1. Available from: http://www.theplantlist.org (accessed 30 Apr 2014).

Townsend, C.C. (1980). Alysseae. In: Townsend, C.C. \& Guest, E (eds.). Flora of Iraq 4(2). Ministry of Agriculture \& Agrarian Reform, Baghdad, pp. 944-988.

Trautvetter, E.R. (1871) Observationes in plantas a dre. Radde anno 1870 in Turcomania et Transcaucasia lectas, nec non in allias quasdam. Trudy Imperatorskogo S.-Peterburgskogo Botanicheskogo Sada [Acta Horti Petropolitani] 1(1): 13-34.

Trautvetter, E.R. (1883) Incrementa florae phanerogamae rossicae. Trudy Imperatorskogo S.-Peterburgskogo Botanicheskogo Sada [Acta Horti Petropolitani] 8(1): 23-268.

Tropicos.org. Missouri Botanical Garden. Available from: http://www.tropicos.org (accessed 30 Apr 2014).

Vassiljeva, A.N. (1961) Cruciferae. In: Pavlov, N.V. (ed.) Flora Kazakhstana [Flora of Kazakhstan] 4. Publishers of Academy of Sciences of Kazakh SSR, Alma-Ata, pp. 171-339.

Walpers, G.G. (1842) Repertorium botanices systematicae 1. Sumtibus Friderici Hofmeister, Lipsiae [Leipzig], 947 pp.

Warwick, S.I., Francis, A. \& Al-Shehbaz, I.A. (2006) Brassicaceae: Species checklist and database on CD-Rom. Plant Systematics and Evolution 259(2-4): 249-258.

http://dx.doi.org/10.1007/s00606-006-0422-0

Willdenow, C.L. (1800) Caroli a Linné Species plantarum exhibentes plantas rite cognitas ad genera relatas, cum differentiis specificis, nominibus trivialibus, synonymis selectis, locis natalibus, secundum systema sexuale digestas 3 . Impensis G. C. Nauk, Berolini [Berlin], III + 2409 pp.

Yurtzev, B.A. (2014) Transberingia Al-Shehbaz \& O’Kane. In: Elven, R., Murray, D.F., Razzhivin, V.Yu. \& Yurtzev, B.A. (eds.) Panarctic flora. V. 1.0. Available from: http://nhm2.uio.no/paf/results?biogeographic=\&bioclimatic=\&region=\&name=Transberingia\#paf6706 (accessed 10 Mar 2014).

Zarqa, N., Kaleem, U.K. \& Rizwana, A.Q. (2013) Draba ishkomania (Brassicaceae), a new addition to flora of Pakistan. Sarhad Journal of Agriculture 29(1): 195-198.

Zhou, T.-Y., Lu L.-L., Yang G. \& Al-Shehbaz, I.A. (2001) Brassicaceae (Cruciferae). In: Wu, Z.-G. \& Raven, P.H. (eds.). Flora of China 8 (Brassicaceae through Saxifragaceae). Science Press, Beijing, and Missouri Botanical Garden Press, St. Louis, pp. 1-193. 